taken to ensure complete extraction before reporting solubilities.

SOLUBII,ITY IN WOOD ALCOHOL,

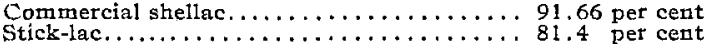

The stick-lac was that referred to, containing 8.5 per cent of woody matter.

I wish to acknowledge indebtedness to my colleague, Mr. J. D. Stuart, who did much of the work incidental to the above investigation.

\section{THE FREEZING POINTS OF MIXTURES OF SULFURIC AND NITRIC ACIDS}

By Walter C. Holmes

Eastern laboratory, E. I. du Pont de Nemours \& Co., Chester, Pa.

Mixtures of sulfuric and nitric acids, commonly designated as mixed acids, are used in enormous a mounts in the chemical industry in nitration processes, as carried out in the explosive and dye industries. Owing to a case where a mixed acid had become frozen during storage, under conditions where freezing seemed improbable, determinations of the freezing points of a large number of mixed acids were carried out in this laboratory. The original object of the work was simply to obtain empirical data regarding the temperatures of freezing for different mixtures. The results, however, were of exceptional interest, as indicating the formation of a definite chemical compound between sulfuric and nitric acids. While thethorough explanation of thephenomena observed was beyond the scope of the work, it is believed that the presentation of these results will be of value as an addition to the knowledge of mixed acids.

The only chemical compound of sulfuric and nitric acids which could be found in the literature is that mentioned by Weber, ${ }^{2}$ who gives it the composition ${ }_{4} \mathrm{SO}_{3} \cdot \mathrm{N}_{2} \mathrm{O}_{5} \cdot 3 \mathrm{H}_{2} \mathrm{O}$, though this composition does not seem proved. The most thorough study of the properties of mixed acids appears to have been carried out by Saposchnikow, ${ }^{3}$ who investigated particularly the vapor pressures, specific gravities, and conductivities of such mixtures in all proportions.

\section{EXPERIMENTAL}

Three sets of mixed acids were made up of roo, 95 and ro3 per cent total acidities, respectively, so that in each set of acids the only variables were the sulfuric acid and the nitric acid contents. The nitric content of the mixtures was varied from zero to approximately 50 per cent in the $r 00$ and 95 per cent mixtures, and up to about 30 per cent in the ro3 per cent mixtures. In all cases the compositions of the mixtures were determined by careful analysis.

The freezing was carried out in test tubes of about one inch diameter, the thermometer being used as stirrer. The test tubes were immersed in an insulated bath. The freezing mixture consisted of ether and carbon dioxide snow, by means of which tempera-

1 Read at the 59th Meeting of the American Chemical Society, St. Louis, Mo., April 12 to $16,1920$.

Ann. Phys. Chem. (Pogg.), 142 (1871), 602.

s $Z$. physik. Chem., 49, 697; 51, 609; 53, 225 (1904-1905). tures as low as $-72^{\circ} \mathrm{C}$. were obtained. Where possible, a standardized mercury thermometer was used, but at extremely low temperatures a toluene-filled thermometer which had previously been compared with the mercury thermometer, was used. Extreme

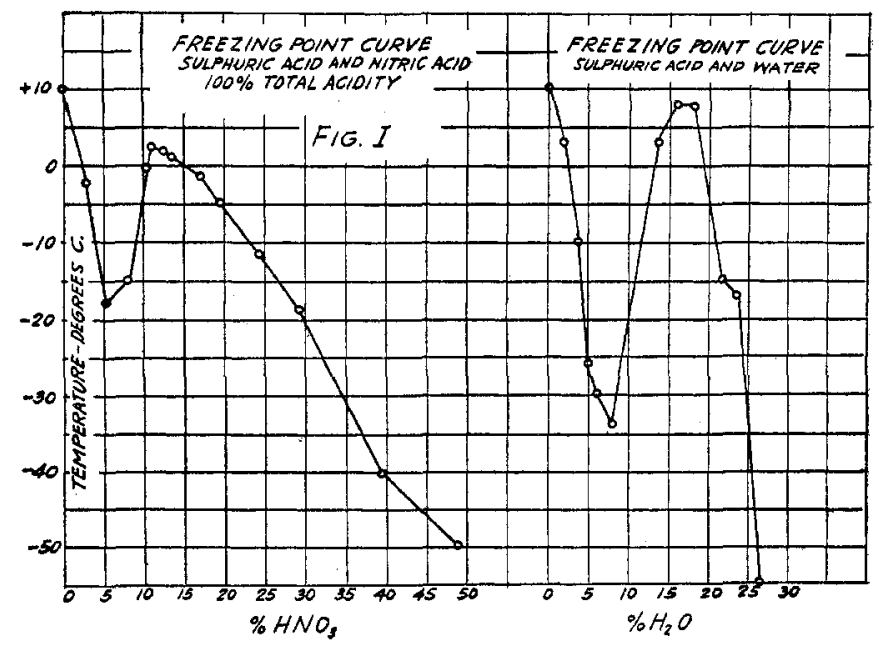

supercooling was met with, one case occurring where the acid did not freeze at a temperature over $60^{\circ} \mathrm{C}$. below the true freezing point, as subsequently determined. As a rule, however, solidification could be induced by inoculation of the sample with a small crystal of frozen acid, care being taken that this crystal should be from an acid of similar composition. Precautions were also taken that, in the final determinations on an acid, the surrounding bath should not be more than a few degrees lower than the freezing point of the acid.

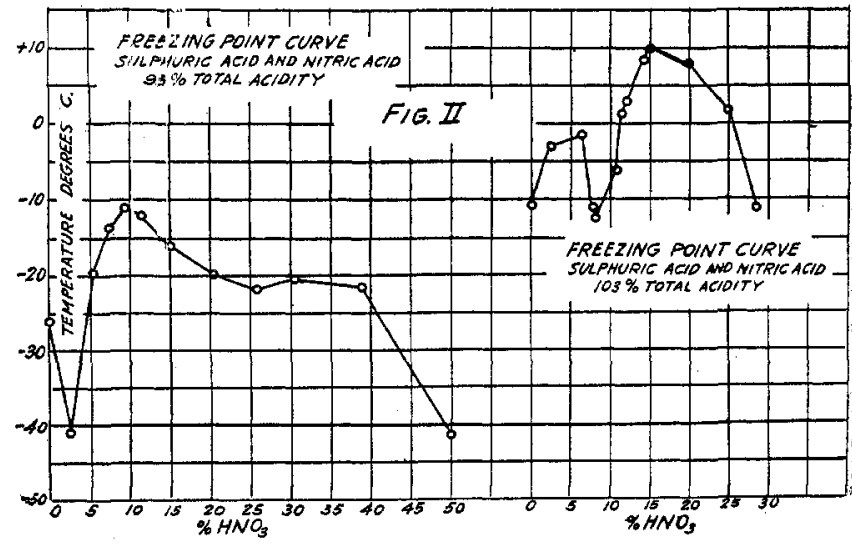

The exact procedure was as follows: A sample was frozen by cooling below the true freezing point and by subsequent inoculation or vigorous stirring, as the case demanded. The temperature of freezing was noted, this temperature being taken at the highest point reached, as in all cases a rise in temperature took place at the time of solidification. The bath was then regulated to not more than $4^{\circ}$ or $5^{\circ}$ below the temperature recorded, and a second sample was frozen. This procedure was repeated until the highest point at which an acid would freeze had been found and checked several times. 
The freezing point values given in the table were obtained by this method:

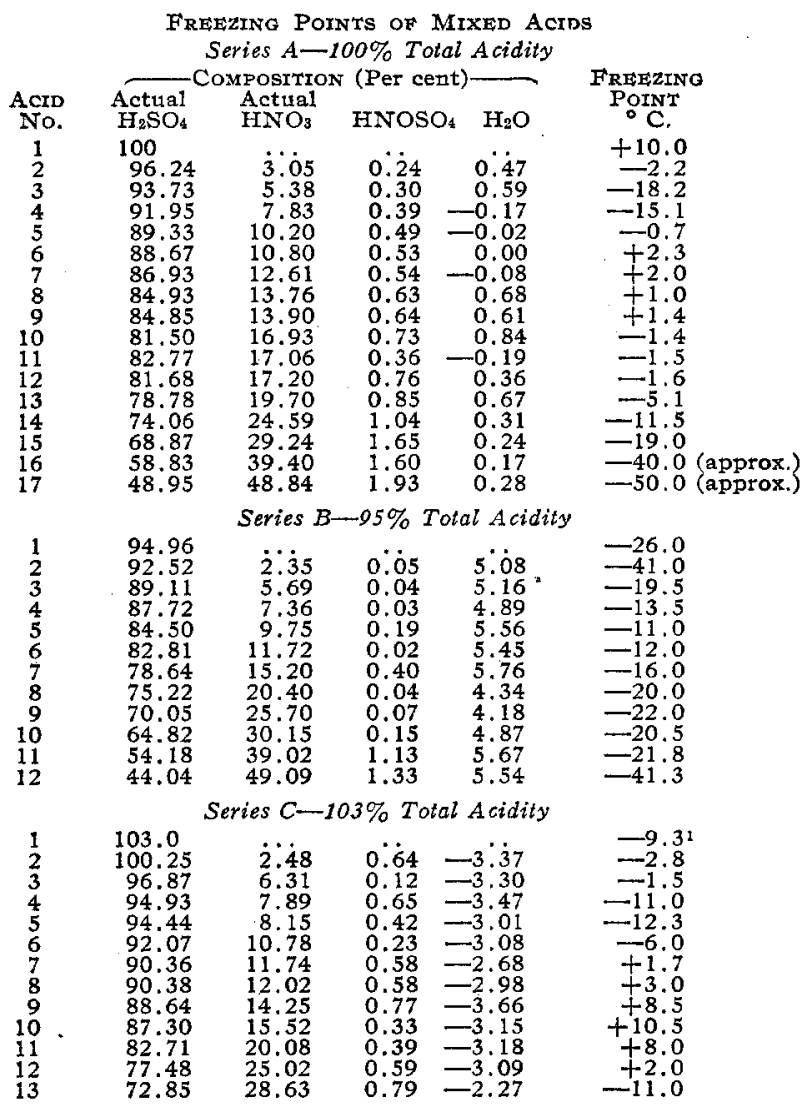

1 The freezing point given, $-9.3^{\circ}$, is interpolated from the table of Knietsch, Ber., $\mathbf{3 4}$ (1901), 4100 . At the time this work was being carried out, no fuming sulfuric acid free from small amounts of nitric acid was available.

\section{DISCUSSION OF RESULTS}

The results tabulated above show that the addition of nitric acid first depresses the freezing point of sulfuric acid. On addition of further amounts of nitric acid, a rapid rise in the freezing point takes place, until a maximum is reached. A general similarity is seen between the freezing-point curves for the three sets of acids. The curves for the mixtures containing 95 per cent and 103 per cent total acidities (Fig. II), however, are somewhat complicated by the presence of a third component, water in the one case and free sulfur trioxide in the other. Two maxima occur in each curve. For this reason, no attempt has been made to explain the form of these curves.

A marked similarity, moreover, is seen to exist between the freezing-point curve of sulfuric acid and nitric acid ( 100 per cent total acidity) and that of sulfuric acid and water (Fig. I).$^{1}$ In the latter case, the curve falls to a minimum of $-34^{\circ} \mathrm{C}$. when 8.4 per cent water is present; then rises to a maximum of $+8.0^{\circ} \mathrm{C}$. with a water content of $\mathrm{I} 5.5$ per cent. In the case of sulfuric acid and nitric acid, the minimum comes at $-18.2^{\circ} \mathrm{C}$., with a nitric content of 5.5 per cent, and the maximum at $+2.3^{\circ}$, when approximately I I.O per cent of nitric acid is present.

The maximum in the sulfuric acid-water curve represents a definitely known compound between

1 The fzezing-point curve for sulfuric acid and water is based-on data given by Knietsch, Ber, 84 (1901), 4100. sulfuric acid and water, the dihydrate $\mathrm{H}_{2} \mathrm{SO}_{4} \cdot \mathrm{H}_{2} \mathrm{O}$. Reasoning from analogy, it seems probable that the maximum in the sulfuric acid-nitric acid curve also represents a definite compound, between sulfuric and nitric acids, since the addition of either component will depress the freezing point of the mixture. Further evidence in favor of the formation of a definite compound is the fact that it was repeatedly found that inoculation of the acid mixtures with a crystal of sulfuric acid would not induce solidification. The use of a crystal of frozen mixed acid was necessary in almost all cases.

The maximum point in the sulfuric acid-nitric acid curve comes at a point where the nitric acid content is ro.80 per cent. It is significant that Saposchnikow found the density of such mixtures to attain a maximum when the nitric content was between ro and I2.5 per cent. The acid mixture having a nitric content of Io. 8 per cent represents almost exactly the combination ${ }_{5} \mathrm{H}_{2} \mathrm{SO}_{4} . \mathrm{HNO}_{3}$.

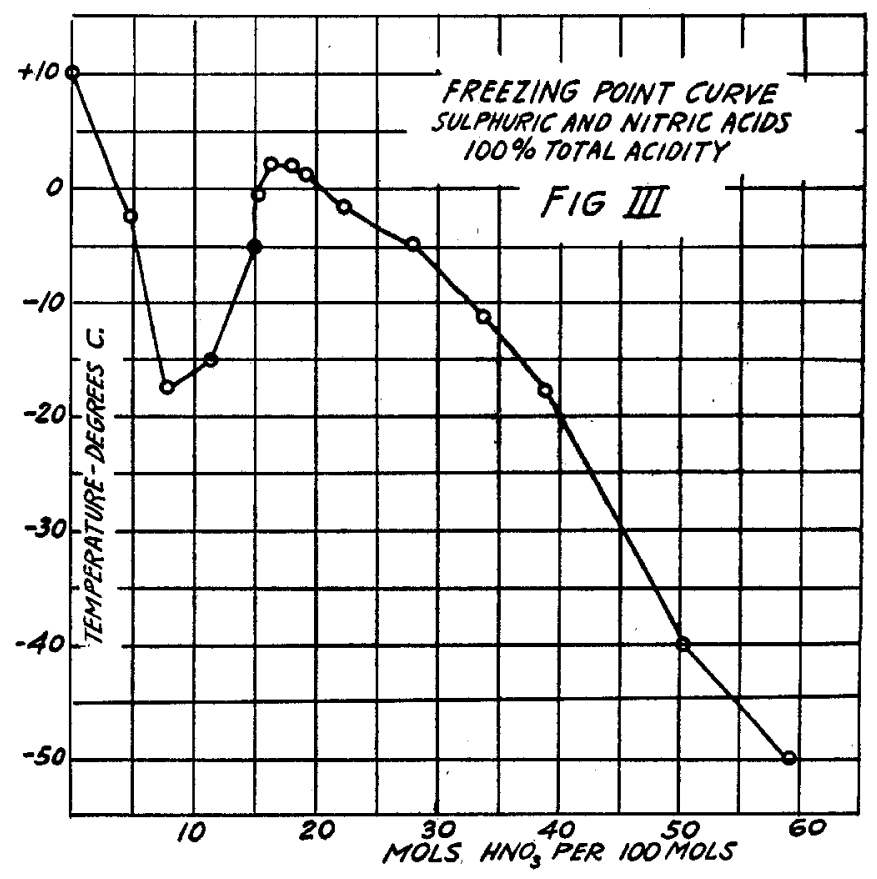

In the freezing-point curve for sulfuric acid and water, while the maximum comes at the composition $\mathrm{H}_{2} \mathrm{SO}_{4}$.$\mathrm{H}_{2} \mathrm{O}$, the preceding minimum comes at the composition $\mathrm{H}_{2} \mathrm{SO}_{4^{-1}} / 2 \mathrm{H}_{2} \mathrm{O}$. It may be a coincidence, but it is at least an interesting one that in the sulfuric-nitric acid curve the maximum comes at the composition ${ }_{5} \mathrm{H}_{2} \mathrm{SO}_{4}, \mathrm{HNO}_{3}$ and the preceding minimum at the approximate composition $5 \mathrm{H}_{2} \mathrm{SO}_{4}^{-1 / 2} \mathrm{HNO}_{3}$.

Saposchnikow did not reach any conclusion as to whether a compound was formed between sulfuric and nitric acids. It was his belief, however, that in mixed acids with a sulfuric content higher than 80 per cent, the nitric acid was dehydrated, with formation of nitric anhydride. This view of Saposchnikow, regarding the condition in which nitric acid is present in mixed acids, is questioned by Marshall. ${ }^{1}$

\footnotetext{
1 "Explosives," 2nd Ed., 1917, p. 123.
} 


\section{SUMMARY}

I-Freezing-point determinations were made on three sets of mixtures of sulfuric and nitric acids, having total acidities of 100,95 , and 103 per cent, respectively, and nitric contents varying from zero to 50 per cent.

2-The freezing-point curve for the Ioo per cent acidity mixtures was found to reach a minimum when the nitric acid content was 5.4 per cent. The maximum was attained with a nitric acid content of 10.8 per cent. The curve showed a marked resemblance to that of sulfuric acid and water.

3-It seemed probable that the composition, at the above maximum, represented a definite chemical compound between sulfuric and nitric acids, the acids at that point being present in the proportion ${ }_{5} \mathrm{H}_{2} \mathrm{SO}_{4}$ $\mathrm{HNO}_{3}$.

4-The freezing-point curves for the 95 and 103 per cent mixtures were complicated by the presence of a third constituent. No attempt, therefore, was made to explain them.

\section{HYGROSCOPICITY OF BY-PRODUCT COKE ${ }^{1}$} By W. A. Selvig and B. B. Kaplan

Fuelis Chemical Laboratory, Pirtsburgh Experiment station, Bureau of Mines, Pittsburgh, Pa. Received April 9, 1920

The experiments described in this paper were undertaken in order to obtain data as to the amount of moisture absorbed by dry pulverized coke on exposure to air of varying humidity in connection with methods ${ }^{2}$ used in many commercial laboratories for the analysis of coke.

In these methods the sample for total moisture determination is taken from the regular sample for analysis by reserving one or more rejected quarters after the sample has been crushed to half-inch size. Moisture is determined in Io lbs. or more by drying to approximately constant weight at a temperature of I0 $5^{\circ} \mathrm{C}$. The laboratory sample for analysis is prepared by crushing and reducing the regular sample until about one pound of 40 -mesh material remains. A sufficient amount of this $40-$ mesh material is transferred to a bottle and dried for one hour at $105^{\circ} \mathrm{C}$. No moisture determination is made in the laboratory on the dried material, it being assumed that the coke will not absorb appreciable amounts of moisture from the air. It is evident that, if the pulverized sample of coke did absorb appreciable amounts of moisture between the time of drying and the weighing of the various portions for analysis, the analysis would be in error corresponding to the amount of moisture absorbed. The volatile matter determination, especially, would be too high.

\section{STANDARD MOISTURE DETERMINATION METHODS}

When a high degree of accuracy is desired, the moisture in coke is determined in two stages. The coke is. crushed to one-half or one-quarter inch size and air-dried to approximately constant weight at a temperature of $30^{\circ}$ to $35^{\circ} \mathrm{C}$. The air-dry sample is

1 Published by permission of the Director of the Bureau of Mines.

2 United States Steel Corporation, "Methods for the Commercial Sampling and Analysis of Coal, Coke, and By-products," 1916, p. 18. then pulverized to 60 mesh and the residual moisture determined by heating a one-gram sample for one hour at $105^{\circ} \mathrm{C.} .^{1}$ Experiments made by the Burea ${ }^{2}$ have shown that total moisture in coke can be determined with adequate accuracy $( \pm 0.5$ per cent $)$ for most purposes by simply heating to constant weight a large sample of lump coke, in any convenient oven, or on a stove, hot plate, or steam coil, at a temperature of $105^{\circ}$ to $200^{\circ} \mathrm{C}$.

The standard methods of analyzing coke as given by the American Society for Testing Materials ${ }^{3}$ state that the total moisture shall be determined by drying the entire sample received at the laboratory without any preliminary crushing to constant weight at a temperature of not less than $104^{\circ}$ or more than $200^{\circ}$ C., and that the loss in weight is to be calculated as percentage of moisture which shall constitute the total moisture in the coke as received in the laboratory. The sample for analysis crushed to a fineness of 60 mesh is prepared from the dried coke without any regard to changes in moisture content. Moisture is then determined on the 60-mesh material by heating a one-gram sample of the coke for one hour in a suitable oven at a temperature of $104^{\circ}$ to $110^{\circ} \mathrm{C}$. The moisture obtained on the 60-mesh material is used simply to calculate the determinations made on this material to a dry basis. The total moisture as determined on the lump size sample is taken as the total moisture content of the coke, and the air-dry figures, are then calculated to the "as received" condition.

\section{OUTLINE OF EXPERIMENTS}

Four samples of coke were selected for the tests, one sample of beehive coke and three samples of byproduct coke. These samples had been previously air-dried and crushed to pass through a 60-mesh sieve. The analysis and origin of these samples are given in Table I.

TABI I-AIR-DRIED 60-Mesh COKE SAMPLas USED IN Tests

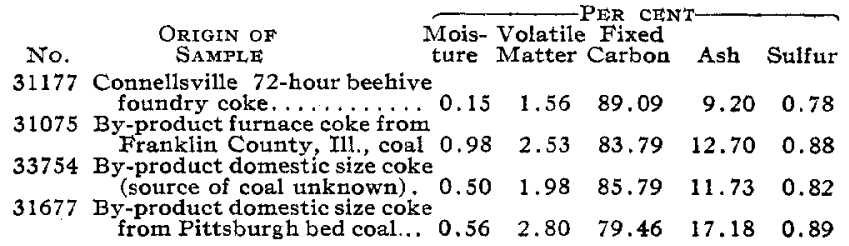

One-gram samples of the 6o-mesh coke were weighed in duplicate into porcelain capsules, dried in an oven for one hour at $105^{\circ} \mathrm{C}$., cooled over sulfuric acid in desiccators, and weighed, the loss in weight being recorded as moisture. In order to prevent any changes in moisture during the weighing of the samples, all weighings throughout the test were made by placing the porcelain capsules containing the samples in glass weighing dishes provided with tightly fitting ground caps.

The uncovered crucibles and contents were placed in one-quart Mason jars containing mixtures of sul-

1 F. M. Stanton and A. C. Fieldner, Bureau of Mines, Technical Paper 8 (1913), 7.

2 A. C. Fieldner and W. A. Selvig, Ibid., 148 (1617), 14.

s Am. Soc. Test. Mat. Standards, 1919, 711 . 